\title{
Le lien fraternel dans la groupalité familiale: séparations et catastrophes de symbolisation
}

\author{
Pascal Roman \\ Martine Drevon \\ Université Lumière, Lyon 2 \\ Isabelle Orgiazzi Billon-Galland \\ Michèle Chappaz \\ Université P. Mendès-France, Grenoble 2
}

\begin{abstract}
RESUMÉ
Les auteurs proposent de considérer la dynamique familiale et les enjeux de transmission au sein du groupe familial à partir de la question du lien fraternel dans un contexte de séparation. Après un rappel des théories fondatrices du groupe et de la groupalité familiale dans le champ de la psychanalyse, quelques repères seront proposés en appui sur les méthodes projectives (Rorschach, C.A.T, T.A.T): se dégageront ce que les auteurs nomment des "catastrophes de symbolisation", dont l'émergence au décours des épreuves projectives témoigne de failles dans la dynamique de transmission au sein de la famille. Enfin, une illustration clinique, à partir de deux situations de fratries, permet de mettre à l'épreuve de la clinique les propositions théoriques et méthodologiques.
\end{abstract}

Mots clés: famile; lien fraternel; symbolisation.

\begin{abstract}
Fraternal bonding in family groups: separations and symbolisation's catastrophes

The authors propose consideration of family dynamics and the problems of transmission in the family group starting from the question of fraternal bonding in the context of separation. After a presentation of fundamental of family grouping in the field of psychoanalysis, some indicators will be proposed with respect to projective methods (Rorschach, C.A.T, T.A.T): and will make clear what the authors designate as "disasters of symbolization" in projective methods and will be described as guide points revealing rifts in the family transmission dynamic. At the end of this paper, clinical illustration is given through two fraternal cases which allows testing of theoretical and methodological propositions of this work.
\end{abstract}

Keywords: family; fraternal bond; symbolization.

Le groupe familial se constitue à partir de l'élaboration des liens entre les différents participants à la dynamique familiale: ces liens, qui se traduisent dans la réalité par des prises de position en termes de comportements - décrits par certains sous la forme de systèmes familiaux - mettent en jeu, au plan intrapsychique, les modalités de transmission entre les générations.

Le groupe fraternel, comme entité au sein du groupe familial, possède au titre de la transmission entre les générations une place singulière.

Bourguignon (1999), dans l'introduction de son ouvrage Le fraternel, met l'accent d'une part sur la dimension paradoxale qui sous-tend la figure du fraternel ("l'autre semblable, intime et étranger") et d'autre part sur la manière dont le fraternel est structuré par et/ou structure le lien familial à partir de l'inscription dans la temporalité, avec l'ordre des naissances, de la différence des sexes au sein de la fratrie et de la taille de celle-ci, représentée par le nombre d'enfants.
- e présent texte présentera cette problématique du lien fraternel selon deux axes:

- un axe théorique, principalement orientée dans la perspective d'une approche psychanalytique $\mathrm{du}$ groupe familial au travers des épreuves projectives,

- et un axe clinique, qui présentera, dans un souci d'illustration clinique, deux situations de fratries, particulièrement envisagées à partir de leurs productions projectives (Rorschach et T.A.T)

\section{1 - LE LIEN FRATERNEL À L'ÉPREUVE DES MÉTHODES PROJECTIVES}

\section{GROUPE FRATERNEL ET GROUPE FAMILIAL}

Si nous ne pouvons que souscrire à la proposition de Bourguignon (1999), qui signale de manière forte une mise au travail des enjeux narcissiques dans le groupe fraternel, cette proposition nous paraît devoir 
être enrichie d'une pensée de la place de la groupalité psychique dans le déploiement des investissements tant narcissiques qu'objectaux.

Pour notre part, et suivant ainsi les travaux issus d'une clinique psychanalytique de la famille, nous soutenons que le lien fraternel, au sein du groupe familial, mobilise des enjeux qui ont à voir avec l'histoire des transmissions, qui concernent tout à la fois la dimension de la génération et la dimension de l'alliance.

Cette question de la double inscription dans des liens de génération et d'alliance, nous la posons ici à partir d'un recours au dispositif de la méthode projective, dans la mesure où, participant des méthodes cliniques, le dispositif de la méthode projective se propose comme butée au regard des processus de symbolisation et de l'histoire de ces processus (Roman, 1997a) qui engage la place du sujet singulier au coeur des liens familiaux.

Une approche familiale des épreuves projectives a été précédemment explorée en appui sur le concept d'appareil psychique familial proposé par Ruffiot (1990). Dans le cadre d'un dispositif de passation familiale (Orgiazzi, 1993, 1994), la pertinence d'une clinique de la transmission entre générations a été mise en évidence, à partir de l'analyse des productions de l'enfant au Rorschach et au T.A.T en référence aux productions de chacun des parents.

Ici, il s'agira de proposer une lecture des enjeux de transmission inter-générationnels et trans-générationnels au travers des protocoles de projectifs individuels. Notre ambition est ici de référer les problématiques de transmission à des productions individuelles-groupales, dans la mesure où des protocoles de fratries ont fait l'objet d'un travail clinique qui vise à affiner une modélisation des indicateurs spécifiques, dans les épreuves projectives, des traces du négatif dans la transmission. La pertinence d'une telle mise au travail de productions individuelles-groupales tient à la fonction psychique singulière de la fratrie au sein de la famille: ainsi que le souligne Jäitin (1998), la fratrie constitue une forme de groupalité psychique familiale, siège du maintien d'une intégrité narcissique de base, au regard d'une non-fiabilité des repères parentaux. À ce titre, la fratrie se trouve porteuse, de fait, des échecs de la transmission au sein du groupe familial.

Le choix de mettre spécifiquement au travail la dimension des restes de la transmission, comme restes à symboliser, participe des choix méthodologiques: autant il nous semble difficile de faire apparaître, en plein, des contenus de transmission, autant il nous semble pertinent d'interroger ce qui se présente en creux sous la forme de ratés de la transmission.

L'hypothèse fondatrice de ce travail tient dans le fait que le groupe familial contient les opérations de symbolisation individuelles, dans la chaîne de transmission qui se déploie, de manière inconsciente, entre les générations. Tout échec dans la contenance de cette transmission se traduit, selon nous, par une marque repérable dans la production du sujet singulier: le dispositif de la méthode projective, dans la mesure où il force les opérateurs de symbolisation, permet un accès privilégié à ce qui se trouve en défaut d'être symbolisé, de ce qui vient faire rupture au sein de l'histoire des transmissions.

Nous proposons de nommer ces ratés de la symbolisation catastrophes de symbolisation ${ }^{1}$, dans le sens où se trouvent comme précipités, dans une figuration traumatique, les opérateurs de symbolisation, tant dans le registre de la différenciation que dans celui de la liaison.

Ainsi, notre travail s'intéressera-t-il aux prolongements groupaux-familiaux des problématiques individuelles de chacun des enfants d'une même fratrie, selon une double entrée:

- celle de la place de l'individu au sein de la groupalité familiale;

- celle de la place du groupe dans la psyché du sujet singulier.

Notre étude sera centrée sur ce qui n'a pas pu être symbolisé dans la génération précédente ou dans les générations précédentes: nous aborderons cette question à partir du repérage de la qualité des liens de filiation tant sur le versant maternel que paternel.

\section{LE NÉGATIF TRANSGÉNÉRATIONNEL}

Le projet est alors de repérer, au travers des protocoles d'épreuves projectives - Rorschach et T.A.T ou C.A.T - d'une part ce qui fait trace de la transmission entre les générations, en plein ou en creux, et d'autre part ce qui se trouve en travail de l'histoire des générations précédentes, voire en panne d'une élaboration et qui se traduit, dans la clinique, sur le mode de la rupture ou de l'effacement. et, au niveau des projectifs, par des catastrophes de symbolisation.

Le contexte de la fratrie autorise un accès à ce que l'on pourrait nommer une zone commune de l'appareil psychique familial, espace d'indifférenciation fondant une enveloppe psychique familiale, support de l'enveloppe généalogique (Granjon, 1987). Dans le même temps, la configuration de la fratrie met à jour les enjeux du maintien a minima d'une intégrité de l'enveloppe généalogique, dans les situations où celle-ci se trouve menacée par une défaillance parentale, en particulier dans les situations de séparation et/ou de placement: Jäitin (1998) montre bien de quelle manière l'identité fraternelle peut venir pallier les risques d'une désagrégation familiale, dans un double mouvement:

- mouvement de restauration de l'intégrité personnelle, dans une remise en question de l'expérience de séparation-individuation,

- mouvement de lutte contre la dépression, qui organise, sur un mode narcissique, un recours $\mathrm{au}$ protogroupe au travers d'un lien fraternel syncrétique (abrasement de la différence des sexes, des générations et des cultures).

Chaque être humain, hérite, quand il vient au monde, d'un négatif transgénérationnel, ce négatif est inconnu, inconnaissable par essence, il se transmet enveloppé, en quelque sorte dans des contenants qui 
permettent de le gérer et de désamorcer son versant dangereux. Ces contenants font partie de la culture familiale. Or, dans certaines familles, ils font défaut, les liens d'alliances et de filiation se trouvant désorganisés. C'est l'observation des processus de la transmission psychique qui met l'accent sur l'arrièrefond inter-subjectif voire trans-subjectif de la vie psychique individuelle. Les membres de la famille, les enfants, auront du mal à s'individualiser, à construire un ancrage filiatif et une identité. C'est dans cette perspective que nous nous intéresserons à la fratrie.

\section{CATASTROPHES DE SYMBOLISATION EN CLINIQUE PROJECTIVE}

La notion de catastrophe de symbolisation vise à rendre compte des ruptures dans le travail de la symbolisation, et de la marque de ces ruptures dans l'histoire de ce travail, et particulièrement dans l'histoire des transmissions entre générations.

Nous nous référons ici à une acception de la symbolisation engagée dans la rencontre avec les épreuves projectives telle qu'elle est proposée par Roussillon (1997), au travers d'un processus qui contient les différentes opérations concourant au système représentatif, travail de symbolisation progressif de la perception (p. 30).
Dans ce contexte, Roussillon propose de décrire le travail projectif en tant qu'il s'établit dans un entredeux de la perception (à partir du stimulus de l'épreuve) et conscience (dans l'énoncé de la réponse) autour de quatre temps distincts (voir tableau 1):

- le temps somatique, qui correspond à la réception des stimuli au niveau corporel et qui conditionne une première organisation au sein d'une logique biologique,

- le temps pré-psychique, caractérisé par la traversée du $\mathrm{Ca}$, concourant à une première organisation, en fonction des modalités d'investis-sement du monde par le sujet et en fonction des données de son histoire et de sa préhistoire personnelle,

- le temps de la métabolisation perceptive, dans le champ de la symbolisation primaire, qui se caractérise par l'affectation du Moi par la perception dans son organisation et dans ses investissements: il s'agit d'un temps d'autoinformation qui inscrit la perception dans un contexte narcissique,

- enfin, le temps de la symbolisation secondaire de la perception, dans des tentatives de liaison préconscientes au travers de représentations de mot.

Tableau 1: Travail projectif et symbolisation

Temps somatique

Temps pré-psychique

Temps de métabolisation perceptive

Temps de symbolisation secondaire
Le stimulus de la planche affecte l'organisation somatique par la voie perceptive

La perception "traverse" le Ca, fonds pulsionnel porteur des traces de l'histoire individuelle et familiale du sujet

Première organisation de la perception et auto-information du sujet

Mise en jeu de liaisons pré-conscientes et mobilisation de représentations de mots, traduites en langage
Dans ce contexte, les catastrophes de symbolisation visent donc à mettre en évidence des éléments qui se réfèrent tout à la fois à l'inscription historique du déploiement des processus de symbolisation (temps pré-psychique, interrogeant les transmissions inconscientes), et aux conditions d'émergence des représentations et de construction de celles-ci dans le contexte de la constitution des imagos (temps de métabolisation perceptive et temps de symbolisation secondaire), dans la double dynamique narcissique-identitaire et objectale-identificatoire.

Nous présenterons ici les différentes formes de catastrophes de symbolisation, regroupées en quatre figures principales, dont on peut repérer la trace au décours des épreuves projectives:

- la première figure se présente sous forme de l'irruption de perceptions immédiates, centrées sur la dimension perceptive selon des modalités sensorielles diverses, voire l'irruption de réponses que l'on peut qualifier d'objets bruts, au sens où ils ne se trouvent pas inscrits dans la chaîne associative,

- la seconde figure tient dans des effets de blanc, qui se manifestent soit sur le versant perceptif (silences, vide représentatif...), soit dans une inscription au coeur des productions verbales par une référence à un implicite, à un non-dit... signifiant un trou dans la chaîne de la transmission,

- la troisième figure tient dans ce que l'on peut qualifier d'îlots de déni, manifestant un défaut d'historicisation des processus de symbolisation, et se traduisant par des marques de références autocentrées, non inscrites dans une dynamique identificatoire, 
- enfin, la quatrième figure se spécifie par une effraction de la fonction contenante, en lien avec un défaut de contenance du négatif de la transmission: l'expression de cette dernière figure se lit de manière privilégiée dans la qualité du lien instauré avec le clinicien et renvoie à la place des imagos dans la dynamique de la transmission entre les générations.

Tableau 2: Catastrophes de symbolisation dans les épreuves projectives

Perceptions immédiates

Effets de blanc

llots de déni

Effraction fonction contenante
Primat du sensoriel

"Objets bruts"

Silences, vides représentatifs

Non-dit, "trous" dans la chaîne associative

Défaut d'historicisation

Références auto-centrées

Lien au clinicien

(rupture vs collage)

Imagos fragiles et/ou instables
C'est la mise à l'épreuve de cette première modélisation des effets de rupture de symbolisation dans la confrontation aux différents protocoles d'épreuves projectives de fratrie qui devrait permettre une élaboration des processus de transmission au sein de la groupalité familiale.

Ainsi, au travers d'un certain nombre d'indices, se référant tant aux catastrophes de symbolisation qu'aux modalités d'élaboration des imagos maternelle et paternelle, les deux mouvements de restauration narcissique et de lutte contre la dépression tenteront d'être mis à jour, en ce qu'ils témoignent de la qualité des liens à l'intérieur de la fratrie: l'hypothèse d'une fonction singulière de l'un des membres de la fratrie, comme porte-souffrance du lien familial (manifesté dans une figure de lien déchiré) traversera en filigrane notre élaboration.

L'hypothèse générale de ce travail tient donc dans une double articulation, dans le registre de l'intrapsychique et de l'intersubjectif:

- les troubles de la représentation, en lien avec les ruptures de la chaîne associative, manifestées par des catastrophes de symbolisation, témoigneraient d'une effraction des contenants du négatif,

- les identifications transmises, au lieu d'aider l'individu à se structurer, peuvent s'avérer paralysantes voire mortifères, notamment lorsque l'un des membres est présenté comme porte-souffrance du lien familial.

\section{2 - CLINIQUE DU LIEN FRATERNEL}

\section{LIENS, GÉNÉRATIONS ET SYMBOLISATION}

Nous avons choisi ici de travailler à partir du matériel clinique issu de la rencontre, dans le cadre d'un mandat judiciaire ${ }^{3}$, de deux fratries, l'une que nous nommerons la fratrie Hercule, avec Octave et Estelle et l'autre la fratrie Épicure, avec Elsa, Samuel et Victor.

Avant d'aborder une lecture des protocoles d'épreuves projectives - Rorschach et C.A.T pour les enfants Hercule, Rorschach et T.A.T pour les enfants Épicure -, nous présenterons quelques éléments de l'histoire clinique de chacune des familles.

Pour la fratrie Hercule, le signalement judiciaire est réalisé par le médecin responsable du Centre de Jour dans lequel Octave est accueilli en soin. Le signalement fait suite à un accident dont a été victime, sa soeur, Estelle, qu'il aurait poussée par la fenêtre du rez-de-chaussée de la maison maternelle. L'idée d'un danger a émergé, idée qui a conduit à une démarche de protection aussi bien à l'égard d'Estelle que d'Octave, ce dernier se trouvant, a contrario, marqué du saut d'un indicible familial, révélant la crise d'une organisation familiale dans ses enjeux de transmission transgénérationnel.

Le contact avec la mère s'établit sur une tonalité mortifère (elle se présente au premier entretien avec un album de photos lui permettant de présenter ses enfants sans avoir à les déranger...) mettant en question la qualité du lien vital au sein de la lignée des générations.

La mère a élevé seule les enfants, le père étant toujours resté vivre à des centaines de kilomètres. Il serait décédé dans un accident de la circulation (mais l'indicible autour de ce décès, qui remonte à plusieurs années, nous évoque une mort par suicide...) alors qu'Estelle était âgée de deux mois et Octave de 17 
mois. Le décès de ce père est présenté de manière banalisée, en revanche, le décès du père de Madame Hercule est relaté avec une charge affective sensible, particulièrement en référence à Octave. Ce dernier était alors âgé de trois ans, Estelle ayant, elle, un peu plus de 18 mois. Cette disparition est présentée comme point originaire des troubles du comportement d'Octave.

$\mathrm{Du}$ parcours de vie des enfants, nous retiendrons de nombreux changements scolaires, les prises en charge d'Octave étant mobilisées sur le versant éducatif et psychique, celles d'Estelle plus sur le versant du somatique, nous les avons pensées comme demande de psychisation d'un désaccordage originaire, en rapport avec un désétayage intergénérationnel.

En ce qui concerne la fratrie Épicure, la saisine du juge des enfants prend appui sur des difficultés financières et éducatives de Madame Dirallou, mère des trois enfants Épicure, difficultés rapportées auprès de l'Assistante Sociale de secteur.

Le couple parental est séparé depuis plusieurs années. Le couple des parents, avant la séparation, travaille comme exploitants agricoles dans les alentours d'une agglomération: il semble s'être construit dans un lien d'alliance en faux-self, basé sur une référence écologiste commune.

La précarité des liens au sein de la famille, mise en évidence au décours du mandat judiciaire, peut être relevée sur plusieurs plans:

- d'une part en termes de carences éducatives, chacun des enfants se trouvant, à sa mesure, dans une situation d'abandon - à cet égard, le lien au sein de la fratrie ne semble pas en mesure de contenir les angoisses archaïques,

- d'autre part, on observe une défaillance de la parentalité, que l'on peut lire autour de la difficulté de chacun des parents à assurer une stabilité dans le lien parental: la mère, suite à la séparation du couple, présentée comme subite, va traverser un épisode dépressif qui la conduira à un départ en Afrique, départ qui se traduit par un abandon des enfants au père (Victor a alors 3 ans); le père, avec sa nouvelle épouse, va, au fil des mois, se trouver débordé par l'éducation de ses enfants en plus des trois enfants de cette dernière et d'un enfant né de leur union: Elsa, Samuel et Victor demanderont alors à rejoindre leur mère et, après quelques va-et-vient entre père et mère, seront désinvestis dans le lien paternel qu'ils interpellent d'ailleurs avec insistance,

- enfin, la place particulière de Victor mérite d'être signalée dans la mesure où il apparaît à la fois comme objet d'une qualité singulière d'investissement au sein du lien familial, et comme porte-souffrance au sein de la fratrie: sa revendication paternelle, ainsi que ses crises clastiques témoignent chacune à leur place d'un enjeu spécifique.

\section{APPORT DES ÉPREUVES PROJECTIVES}

Le matériel clinique recueilli auprès de ces deux fratries sera travaillé successivement, dans le fil des problématiques dominantes des protocoles d'épreuves projectives.

Pour la famille Hercule, l'accent sera mis d'une part sur les modalités d'inscription généalogique dEstelle et Octave et d'autre part sur la spécificité des troubles de la représentation.

Pour la famille Épicure, l'analyse du matériel sera construite autour des modalités de transmission dont chacun des enfants va se trouver porteur de manière spécifique.

\section{Famille Hercule}

La question des imagos, dans ce qu'elle signe de l'inscription dans la généalogie, ainsi que le repérage des troubles identitaires constitueront les deux axes de ce premier temps d'analyse.

\section{L'inscription dans la généalogie}

Le premier axe de travail concerne la constitution des imagos et le travail des identifications.

Chez Octave, le fonctionnement psychique est majoritairement marqué par la passivité, l'inhibition, l'appauvrissement fantasmatique et le vide interne, dans une tentative d'intériorité qui se trouve plaquée ("il réfléchit", planche 3, C.A.T), ou qu'un recours à la réalité de la relation au clinicien tente de ranimer. Aucune différenciation, tant sexuelle que générationnelle, n'est possible dans ce vide interne (C.A.T, planche 2), vide dont rend compte la représentation d'une chambre vide que seules viennent peupler des peluches (C.A.T - 5): la recherche de ce contact régressif et infantile va échouer dans une groupalité indifférenciée (C.A.T - 5, 6 et 7).

C'est la défaillance de sa capacité à contenir, à maintenir permanente une véritable représentation psychique interne qui s'actualise (Rorschach, I: "une chauve-souris / elle avait des ailes"; "un masque / non! pas un masque! parce que ça ressemble à un masque", Rorschach, II: "un papillon il a l'air drôlement écrasé / non j'ai pas dit qu'il était écrasé”) Octave ne peut alors se situer que dans le refus ou l'agir qui occupent l'espace de ce manque à représenter, évoquant le passage à l'acte à l'égard de sa soeur lorsqu'il se trouve confronté à l'imago paternelle/phallique (planches IV et VI).

Estelle apparaît beaucoup plus dynamique au niveau de ses défenses dans la mise en oeuvre active de défenses maniaques anti-dépressives (C.A.T - 1: "leur maman est partie, ils ont l'air de dire miammiam... ça a l'air bon"), luttant contre une absence d'étayage qui ne peut être comblé (C.A.T - 9: "on dirait que c'est un petit lapin qui est malade...qui essaye de s'endormir" et 10: "la maman du bébé et le petit chien (....) il a l'air de tomber comme ça"). L'immaturité et la parentification au sein d'une relation primaire dominent (C.A.T - 2: le bébé aide la mère, la différenciation des sexes ne se situant en fin de planche qu'à un niveau de repérage sexuel biologique mâle/femelle). L'espoir d'un contact 
sécurisant avec la peluche, observé chez Octave, se retrouve chez sa soeur, dans une dualité mère - enfant dont toute référence paternelle est exclue (planche 5), imago phallique puissante et nantie, immédiatement dénigrée et dévalorisée dans une impossible élaboration triangulée (planche 6).

Le second axe de travail sera centré sur la dynamique identitaire et interrogera particulièrement la question de la perte.

Octave, dans la pauvreté globale de son élaboration pose la question d'un lien à l'autre, dans une recherche de type identitaire (C.A.T - 8: "des singes qui discutent y en a qui discutent sur une chaise et d'autres sur un canapé et ceux qui sont sur un canapé la fille elle boit du thé"). Et c'est son effroi qu'il verbalise, effroi dont il attribue la cause au clinicien ("pourquoi vous me dites ça ?", à voix très basse) dans un mouvement de suspicion envers autrui et des mesures très défendues (banalité, refus). Ce dont il se défend parvient pourtant à filtrer dans des productions pétrifiées et menaçant le vital (Rorschach - VIII: "un fossile...et puis des rats qui tournent autour"), Octave s'avérant porteur de la dimension morbide, acteur d'un contenu dévitalisé qui va peu à peu se déshumaniser ("forme de collier", "fossile", "des formes imaginaires").

Chez Estelle, la relation à l'objet apparaîtra beaucoup plus duelle, voire individuelle et identitaire, que véritablement triangulée (C.A.T - 2: "ce sera pour moi, ce sera à moi (...) on dirait que dans la guerre ils disent oh hisse”), en dépit d'une entrée immédiatement sexualisée au Rorschach. Ce trop d'excitation paraît peu en adéquation avec le refoulement de la latence que l'on serait en droit d'attendre (Rorschach - I: "des hommes qui touchent une dame", II: "c'est des coeurs....ils ont l'air de s'embrasser (...) je vous aime”). Cette surcharge d'excitation pulsionnelle, inélaborable, s'effrite petit à petit, malgré l'aspect défensif positif du départ (puissance interdictrice des policiers). La verbalisation perd de sa cohérence et la pensée dérape dans un processus de désymbolisation avec rabattement dans la réalité ("ils ont l'air d'être au bureau de tabac parce qu'on voit tout de suite le tabouret"). L'attaque des liens de la pensée se prolonge, dans une tentative de repérage identitaire à travers le fonctionnel, qui tient lieu et place d'une ébauche de repérage identificatoire ("des monsieurs" (...) ils ont l'air (...) ceux qui servent dans les hôtels, avec les noeudspapillons (...) le costume”). La référence humaine s'étiole dans une perte de la qualité sexuée de l'imago paternelle, alors passive et attaquée, voire détruite (Rorschach - IV: "ils pendent des sabots", "un monsieur (...) en présentation, avec des crochets...presque un monstre"), dans un glissement sujet - objet mal différencié ("ils pendent des sabots / ses yeux pendent"). Cette impossible élaboration de l'imago masculine/paternelle, passive et sacrifiée, s'avère le pivot central du protocole: à partir de ce moment-là, en effet, la passivité prédomine (planche VI: "un oiseau sur une pierre il regarde comme ça (...) les reflets il est au bord de l'eau"), gelant tout mouvement objectal dans un processus de récupération narcissique s'étayant sur une relation spéculaire à l'imago féminine/maternelle (planches VII et IX: reflets, glace, robe). La problématique d'individuation et de différenciation ne peut aboutir qu'à l'image de l'extrême fragilité et d'inconsistance finale sur laquelle va alors se clore le travail associatif ("et là c'est un bout d'herbe").

\section{Les troubles de la représentation: ruptures de la chaîne associative et catastrophes de symbolisation}

Il s'agit ici de repérer tout spécialement les marques de la discontinuité, de la mise en crise, des opérateurs de symbolisation chez Octave et chez Estelle, particulièrement là où ces ruptures s'inscrivent dans le champ du langage.

On peut en mettre en évidence plusieurs figures qui, chacune à leur manière, concourt à définir tout à la fois des lignes défensives et les supports pour une réorganisation: l'écrasement des représentations, les trous dans l'élaboration représentative, la tentative d'instauration de liens primaires fondateurs et/ou supports de la différenciation.

L'écrasement des représentations se traduit chez Octave par la réduction dans le discours de la fonction d'écart propre à fonder la représentation au Rorschach, en écho au discours de la mère dans le temps de la restitution (I, II), l'écrasement porte sur le processus même de la représentation (VIII), laissant apparaître un doute sur la qualité de l'inscription identitaire. Cet écrasement se donne à voir au C.A.T au travers de la réduction de l'écart propre à fonder l'organisation des représentations au C.A.T avec l'écrasement de la différence intergénérationnelle au C.A.T (en particulier à 4, 5,6 et 10 )

Chez Estelle, l'écrasement des représentations se traduit essentiellement par des confusions, des glissements: glissement de la scène de la représentation à la scène psychique (II), glissement d'une représentation humaine à une représentation monstrueuse (IV), de l'humain à l'animal (V, VII, IX), glissement d'une représentation à une autre (d'une scène à l'autre...X). Au C.A.T, le support figuratif favorise une reprise a minima: l'écrasement des représentations se joue toutefois également sur le registre de la confusion, qui tient lieu d'évitement du traitement du lien, en tant que support de l'élaboration identitaire.

Le trou dans l'élaboration représentative prend forme, pour Octave, au travers de la vacuité de l'élaboration représentative qui s'exprime au travers du vide représentationnel (III), de l'irruption du stimulus sur la scène du somatique (IV). Au C.A.T, c'est dans l'évitement des représentations, l'absence de reconnaissance des liens (indifférenciation, 8 et 10) et la perte de la qualité vitale $(5,6$ et 9$)$ que s'exprime cette carence.

Chez Estelle, les réponses au Rorschach s'inscrivent dans une dimension de leurre au regard du sexuel, leurre fonctionnant comme modalité antidépressive: en tant que telle, la situation Rorschach semble prise dans une séduction qui vient rejoindre une zone traumatique, celle du secret au sein du 
transgénérationnel. Les exemples de la faillite de l'activité représentative ne manquent pas (I, II, III), faillites qui se manifestent au travers de trous dans la chaîne associative, obturant la qualité du sens et renforçant l'instabilité de la rencontre avec l'objet. Les nombreux scotomes au C.A.T (3, 5, 10) supportent la discontinuité de l'inscription dans la lignée

La tentative d'instauration de liens primaires fondateurs et/ou supports de la différenciation s'élabore, pour Octave, à partir d'un accrochage au matériau lui-même (Rorschach IV, VII, VIII - a minima - et IX), au travers de la mobilisation de l'engramme dans un projet de mise en forme (et de l'échec de ce projet). Au C.A.T, la description du matériel, dans sa dimension essentiellement perceptive tente de pallier à l'instabilité des objets internes: le matériel est alors investi pour lui-même, comme support d'un lien primaire qui apparaît comme lien de survie psychique.

Pour Estelle, on pourrait dire que la quête d'un étayage dans le lien primaire ne résiste pas aux défenses anti-dépressives: la faillite de l'organisation des représentations, les ellipses dans le traitement du lien tendent à laisser sans fondement le travail de la différenciation. C'est ici davantage sur l'investissement des fonctions perceptives que se joue la quête d'un lien primaire, au travers d'un recours au voir au Rorschach, recours qui se déplace au C.A.T dans la mesure de l'appui que peut constituer la figuration du matériel: peut-être peut-on dire que le point d'appui du lien tiendrait davantage à la fonction transitionnelle, avec la mention récurrente on dirait.

Tableau 3: Famille Hercule - Synthèse des expressions projectives

\begin{tabular}{|c|c|c|c|}
\hline & PROBLÉMATIQUE & OCTAVE & ESTELLE \\
\hline \multirow{2}{*}{ INSCRIPTION GÉNÉALOGIE } & $\begin{array}{c}\text { IMAGOS } \\
\text { IDENTIFICATIONS }\end{array}$ & $\begin{array}{l}\text { Pas de différencia-tion } \\
\text { sexuelle et générationnelle } \\
\text { Vide interne } \\
\text { Refus, agir }\end{array}$ & $\begin{array}{l}\text { Défenses maniaques } \\
\text { Parentification } \\
\text { Espoir }\end{array}$ \\
\hline & $\begin{array}{l}\text { IDENTITÉ } \\
\text { PERTE }\end{array}$ & $\begin{array}{c}\text { Quête identitaire } \\
\text { Effroi, menace vitale }\end{array}$ & $\begin{array}{l}\text { Relation duelle } \\
\text { Sexualisation lien au féminin } \\
\text { Fragilité identité }\end{array}$ \\
\hline \multirow{3}{*}{$\begin{array}{c}\text { TROUBLES } \\
\text { DE LA SYMBOLISATION }\end{array}$} & $\begin{array}{l}\text { ÉCRASEMENT DES } \\
\text { REPRÉSENTATIONS }\end{array}$ & $\begin{array}{l}\text { Doute sur les repré- } \\
\text { sentations }\end{array}$ & $\begin{array}{l}\text { Glissement des } \\
\text { représenations sur la scène } \\
\text { psychique }\end{array}$ \\
\hline & $\begin{array}{c}\text { TROUS DANS } \\
\text { L'ÉLABORATION } \\
\text { REPRÉSENTATIVE }\end{array}$ & Vide des représentations & $\begin{array}{l}\text { Discontinuité } \\
\text { Scotomes }\end{array}$ \\
\hline & $\begin{array}{c}\text { TENTATIVE INSTAURATION } \\
\text { LIEN PRIMAIRE }\end{array}$ & $\begin{array}{c}\text { Accrochage au matériau } \\
\text { (description) }\end{array}$ & Investissement perceptif \\
\hline
\end{tabular}

\section{Famille Épicure}

La lecture clinique des protocoles d'Elsa, Samuel et Victor nous a conduit à considérer que chacun des trois enfants de la fratrie semblait porteur d'une part de la problématique familiale.

\section{Problématique du lien: la transmission de la lignée maternelle en question}

La lecture du protocole de Rorschach d'Elsa révèle essentiellement une problématique du lien, dont l'impact va s'avérer prégnant tout au long de ses associations. Citons la planche III, qui illustre particulièrement la difficulté de cette grande adolescente à intégrer la nécessité d'une individuation-séparation vis-à-vis de l'imago maternelle:"deux femmes, avec deux foetus, on dirait qu'elles se séparent... là ça fait le cordon”. 
Cette préoccupation autour de l'attachement d'une femme à son foetus atteste tout à la fois de l'entrave que constitue sa position de fille dans le lien de filiation à sa propre mère, tout comme les difficultés à s'enraciner dans une transmission familiale. Le ventre, contenant maternel, est à nouveau évoqué à la planche IX à partir de "gnomes", avec "son nez, une bouche avec le ventre là, gros ventre”... D'après la définition qu'en propose le dictionnaire ${ }^{5}$, les gnomes sont des esprits présidant à la destinée de chacun, êtres contrefaits par leur immuable réduction. L'association qui suit ("une fontaine") renforce encore la prégnance de la question des origines, dans une tonalité dépressive qui se dégage du commentaire ("c'est bleu et ça tombe").

Cette tonalité dépressive perdure et clôt la passation. La planche $\mathrm{X}$ (qui est, à l'épreuve des choix, celle qui est retenue comme étant la préférée, voit la production d'une réponse mettant en scène l'alternance entre la vie et la mort: "tête de pirate, parce que ça fait penser à là où il y a une vie". La pulsion de vie, qui sous-entend la lutte contre la pulsion de mort, est ici associée à une référence masculine qui ouvre quelque peu le protocole, l'imago masculine/paternelle étant relativement absente, très défendue, comme en témoigne la planche IV: "on dirait l'Italie... pfff... je vois rien d'autre, je vois rien d'autre".

\section{Une filiation sexuée douloureuse: la transmission de la lignée paternelle en question}

Tout comme Elsa ouvrant sa production à l'épreuve de Rorschach par une image péjorative et inquiétante, Samuel associe cette première planche maternelle à une image monstrueuse qu'il rejette à l'enquête. Il livre d'emblée une charge agressive avec une réponse "guêpe", représentation féminine nantie, dans un magnifique lapsus de "testicules" (en lieu et place de "mandibules"), posant dès le début de la passation l'ampleur de la problématique sexuelle dont il est porteur.

La revendication phallique (planche I: "un avion, il $y$ a les réacteurs", planche III: "deux femmes, on dirait qu'elles ont de la poitrine", planche X: "la Tour Eiffel") traverse effectivement, de toute évidence, la vie psychique de cet adolescent, tout comme Elsa était préoccupée par le ventre maternel, au sein d'une filiation générationnelle et sexuelle douloureuse.

Comme au Rorschach, le T.A.T. montre les difficultés de ce garçon à s'approprier l'objet phallique comme support identificatoire dans une transmission des générations coûteuse. La planche 1 ("c'est un petit garçon il a son violon et il a marre de jouer du violon...il trouve ça dur...et puis il est fatigué, on dirait qu'on le force à jouer du violon") comme les planches 3 B.M ("c'est une dame qui pleure...elle vient de se faire quitter par son mari...elle est toute seule...elle se demande ce qu'elle va devenir sans lui et puis il y a les enfants à élever") ou 5 ("c'est une dame qui dit à son enfant de se coucher (...) et puis y a son père qui va rentrer et s'il dort pas encore il va l'engueuler") mettent en avant une imago paternelle assez fruste qui n'apparaît qu'à travers un discours stéréotypé et infiltré de contraintes extérieures, posant la question de la qualité des objets internes. La planche 7 B.M. nous montre un modèle paternel à coloration perverse: “c'est le père au mec...qui lui montre, qui lui dit " regarde la fille là-bas elle est bien"...le père essaie de monter une baraque...enfin de mettre son fils avec la fille et lui il veut pas, il la trouve pas jolie", au travers duquel la figure paternelle met en scène une initiation sexuelle agie à la place du fils. Les rôles sexuels et les rôles familiaux (planches 6 et 7 B.M), dans leur aspect éminemment caricatural, témoignent de la conflictualité d'une identification sexuelle insuffisamment intégrée. La fragilité identificatoire empêche une conflictualisation de la relation oedipienne, comme à la planche 8 B.M dans laquelle au lien père/fils se substitue un lien de couple ("y en a un qui va se faire opérer parce qu'il vient de se prendre une balle, y a sa femme qui est juste à côté... qui est triste") et à la planche 10 , où émerge une relation mère/fille accompagnée d'un lapsus concernant le genre sexuel ("c'est la mère avec sa fille, la fille elle est triste et puis la mère elle est inquiète pour lui").

La différence des sexes et des générations n'est pas clairement intégrée, Samuel ne parvient pas, à la planche 2 par exemple, à établir une triangulation réussie dans la mesure où les imagos parentales ne sont pas réellement différenciées, l'étudiante tout comme l'homme étant dans une contrainte extérieure où le désir de chacun est absent ("c'est des paysans qui cultivent leur champ (...) c'est une étudiante, il faut qu'elle aille à l'école...le paysan il regarde ses champs, il faut qu'il les cultive").

Comme pour Elsa, il se dégage un fond dépressif qui émaille l'ensemble du protocole ("il trouve ça dur il est fatigué on le force à jouer du violon / le paysan il regarde ses champs il faut qu'il les cultive" / "et puis y a les enfants à élever" / "il faut qu'il trouve du travail / la fille elle est triste" ); la planche 13 B corrobore et met en exergue cette tonalité dépressive avec 'c'est un petit enfant qui pense, qu'il pourrait habiter dans une plus belle maison parce que la sienne elle est...enfin il pourrait habiter dans de meilleures conditions, avoir une vraie vie, dans une belle maison, avec un vrai jardin...il est malheureux de vivre ici". L'enfant exprime une souffrance dépressive face à un contenant qui ne le satisfait pas et le frustre, ce qui interroge sur la qualité de l'enveloppe familiale dont il n'a rien à attendre ni à hériter... et ce d'autant plus que Victor, dont nous allons analyser à présent le matériel projectif, développera la même thématique avec encore plus de douleur.

\section{Au risque de se perdre: La transmission parentale en question}

Victor donne l'élaboration suivante à la planche 13 B qui met en jeu la problématique de la solitude: “c'est un petit garçon il...il vit dans la misère, il se pose des questions à propos de tout, lui, sa maison, ses parents...il aimerait bien avoir une belle maison, 
être riche, comme les autres". L'atteinte narcissique s'exacerbe dans un vécu masochiste, faisant écho à la planche 12 ("c'est un coin abandonné, y a jamais personne"), désert relationnel dans lequel Victor ne parvient pas même à se raccrocher au cadre perceptif. La précarité du lien à l'objet peut même déclencher une défense persécutoire comme à la planche 3 B.M (“c'est quelqu'un qui pleure parce qu'il est pauvre...puis tout le monde le rejette puis il pleure, il se sent tout seul parce qu'il est dans un coin"), face au manque d'étayage dans un vécu de carences sans possibilité de dégagement que pourrait fournir un tiers. Le tiers ne peut consoler parce que l'inscription n'a pas eu lieu: l'objet phallique ne peut pas servir puisqu'il est abîmé, irrémédiablement cassé comme en témoigne le travail associatif à la planche 1: "c'est un enfant qui est déçu parce que son violon il est cassé...et parce qu'il sait pas en jouer...il arrive pas à le réparer et puis il pourra jamais savoir comment en jouer puisqu'il pourra jamais le réparer". Cette impossible appropriation de l'objet phallique le place dans l'impuissance fondamentale d'une destinée vouée à la fatalité. L'inscription ne se fait pas dans cette temporalité bloquée, protégeant d'une violence que nous voyons émerger à la planche 2: "c'est une femme qui est en fuite, qui fuit...et des hommes qui frappent quelque chose et une femme qui prie". La transmission générationnelle semble comme rompue, elle aussi cassée à travers le vécu d'un enfant qui ne se vit que comme objet de déception pour l'autre; c'est ce que les planches 6 et 7 B.M mettent clairement en évidence: "c'est la mère elle est déçue par son fils et le fils il est désolé...il est dégoûté de ce qu'il a fait" / "c'est le père il va voir son fils (...) le père il est déçu de son fils".

L'imago maternelle se réduit à "une femme qui semble énervée parce qu'elle fait une tête bizarre et puis on dirait qu'elle va crier", figure négative qui ne parle pas mais crie, en dehors de toute préoccupation maternelle pourtant indispensable comme premier espace de la transmission psychique. Victor ne parvient pas à symboliser la castration ni, comme Samuel et Elsa, à s'appuyer sur une loi paternelle phallique structurante. La planche 8 B.M ("y a des gens qui font un assassinat ils tuent quelqu'un... y en a un c'est le chef, il lui dit de le faire et l'autre il veut pas le faire mais il est obligé...et y a un petit garçon tout innocent il sait pas ce qui se passe, il fait comme si de rien n'était, mais il pleure un peu à l'intérieur") met en scène un petit garçon soumis à l'obligation sadique (sexuelle?) d'un dominant n'aboutissant qu'au retournement sur soi de la charge agressive. L'enfant ne peut s'inscrire dans une lignée qui soutient, abandonné de tous, enfant sacrificiel qui pleure à l'intérieur.

La souffrance de Victor s'avère effectivement très importante et va envahir l'ensemble du protocole de Rorschach. Les représentations qui y sont données s'articulent autour de la prépondérance du masochisme et de ses liens à un sadisme persécutoire que l'on retrouve de manière récurrente de planche en planche: planche I: "un monstre / il paraît gentil mais en fait (...) il montre que cette partie, ses yeux tu vois qu'il est gentil mais ses autres yeux ils sont méchants", planche II: "un dos là / la colonne vertébrale est déchirée" puis "un papillon / ça fait deux ailes (...) et puis ça fait un cour comme s'il était enfermé dans quelque chose (...) ça fait des pauvres yeux, quelqu'un enfermé dans quelque chose...un regard bizarre", planche III: "une tête de mort tenue par deux femmes" / c'est sinistre, vraiment de quelqu'un qu'on dirait sans vie", planche V: "quelque chose de bizarre... vicelard...qui se moque de nous (...) un joli papillon mais c'est les ailes, comme si elles se moquent de nous", planche VI: "un pauvre tigre martyrisé / sa tête elle est écrasée (...) il est tout malade" et enfin, planche VIII: "un dos très très musclé; un dos déchiré / un dos robuste mais c'est déchiré sa colonne, ça tient juste en équilibre, la pyramide, le dos déchirê".

La persévération de la représentation du dos musclé / déchiré (planches IV, VI, VIII et IX) traduit à l'évidence le double mouvement d'une force virile masculine, tout à la fois mise en avant et attaquée; cette barrière musculaire montre le flou des limites intérieur / extérieur ("colonne vertébrale déchirée"), la mauvaise délimitation dedans / dehors (avec les associations "colonne vertébrale déchirée", "dos très très musclê", "une bougie qui fond") révélant l'inefficacité de cette barrière corporelle contre investie face à la fragilité interne.

\begin{tabular}{|c|c|c|c|}
\hline PROBLÉMATIQUE & ELSA & SAMUEL & VICTOR \\
\hline FILIATION MATERNELLE & $\begin{array}{c}\text { Insistance lien-séparation } \\
\text { Dépression }\end{array}$ & Lien maternel "phallique" & Lien persécutoire \\
\hline FILIATION & Evitement imago paternelle & $\begin{array}{c}\text { Imago paternelle perverse } \\
\text { Triangulation difficile }\end{array}$ & $\begin{array}{c}\text { Attaque du masculin- } \\
\text { phallique }\end{array}$ \\
\hline
\end{tabular}




\begin{tabular}{|c|c|c|}
\hline \multirow{2}{*}{ TRANSMISSION PARENTALE } & $\begin{array}{c}\text { Absence de support fiable pour la transmission des imagos } \\
\text { Conflictualisation problématique des imagos }\end{array}$ \\
\cline { 2 - 3 } & \multicolumn{2}{|c|}{$\begin{array}{c}\text { Porte-souffance du lien } \\
\text { familial }\end{array}$} \\
\hline
\end{tabular}

\section{POUR CONCLURE}

Cette approche projective illustre, en premier lieu, une problématique du lien importante tant au niveau des liens d'alliances de couples, des liens de filiation et des liens fraternels. Elle devrait permettre, au-delà de cette première élaboration, de construire une véritable grille de la transmission psychique entre les générations au Rorschach et au T.A.T.

Pour l'heure, ce travail montre combien les identifications transmises, au lieu d'aider l'individu à se structurer, peuvent s'avérer paralysantes, voire mortifères, avec la fonction singulière de l'un des membres de la fratrie, porte-souffrance du lien familial dans sa valence narcissique - Victor et son dos déchiré -, porte souffrance du lien familial violent - Octave et l'agir dans le manque à représenter de la dimension morbide du non-dit familial.

Dans les deux fratries avec lesquelles nous avons travaillé, se dégagent des éléments dépressifs et désorganisationnels qui vont crescendo, s'articulant autour d'une problématique du lien et du maternel pour les filles, Elsa ou Estelle, d'une problématique du masculin et de l'appropriation phallique pour Samuel, et d'une problématique narcissique, voire identitaire pour Victor comme pour Octave.

Notre analyse s'est organisée autour de deux axes:

- celui des imagos paternelles et maternelles défaillantes et de la défaillance parentale, concrétisée par la question de la séparation des enfants du milieu familial dans un cadre judiciaire; le déploiement imaginaire se fonde sur la structure et les contenus de l'imaginaire familial, dont la fratrie constitue une entité spécifique qui condense les enjeux de filiation et d'affiliation,

- celui des troubles de la représentation en lien avec des ruptures de la chaîne associative et des catastrophes de symbolisation.

En ce qui concerne l'enveloppe généalogique, nous étions confrontés à un manque d'éléments de réalité concernant l'histoire des lignées, tant du côté maternel que paternel dans les deux situations présentées. Ne sachant rien, nous ne pouvons parler de ce que nous ne connaissons pas; cependant, si nous-mêmes ne pouvons rien savoir de ce négatif, nous pouvons en connaître les effets.

Nous pouvons, en particulier, parler de ce que cela a produit chez chacun d'entre nous sur le plan contretransférentiel, de ce que cela a produit à travers les projectifs, mais nous ignorons de quoi ce négatif est composé. Il s'agit là de la partie inatteignable de l'inconscient, qui se déploie sur fond d'originaire: c'est alors que nous parlerons du négatif transgénérationnel, négatif dont la dimension déliante et destructrice appelle un contenant.

L'être humain hérite quand il vient au monde, nous l'avons dit, du négatif transgénérationnel. Ce négatif, inconnu et inconnaissable, se transmet dans des formes contenantes qui appartiennent à la culture familiale. Mais que se passe-t-il quand ces contenants font défaut?

L'analyse clinique des protocoles des deux fratries Hercule et Épicure nous permet d'apporter quelques pistes de compréhension quant au destin d'une contenance introuvable du négatif.

Si nous reprenons l'hypothèse d'une effraction des contenants du négatif, pour la famille Hercule, il semble que la mort du père soit venue en écho rompre un équilibre fragile, non fondé sur des liens d'alliances et de filiation, ou plus précisément fondé sur un déni de ces liens.

En ce qui concerne la famille Epicure, les enfants n'ont pas pu faire famille et ils s'en défendent paradoxalement en disant qu'ils n'en n'ont pas besoin, qu'ils se sont fait tout seul. La chaîne qui relie les générations les unes aux autres, et qui les fonde dans un ancrage mythique à l'origine parait être rompue. C'est ce désancrage filiatif qui fait le plus souffrir les enfants et qui les a empêché de se construire une identité tant au niveau intrapsychique qu'intersubjectif.

Dans ces deux cas, l'identité fraternelle, point de condensation entre le familier et le non-familier, tout en se trouvant mobilisée dans une tentative de contenir la souffrance du lien familial, pallie difficilement la désagrégation du lien familial. D'une certaine manière, dans une approche familiale des enjeux de séparation-individuation au sein du groupe familial, on peut reconnaître, au sein de ces deux familles et à des titres divers, une résolution problématique de la position dépressive familiale (Roman, 1999).

Ainsi, en l'absence d'un travail d'élaboration de la souffrance des liens, c'est de la mémoire de l'oubli dont vont hériter les générations suivantes, le travail de transformation restant alors à la charge de ses héritiers. 
Bourguignon, O. (1999). Le fraternel. Paris: Dunod.

Jaïtin, R. (1998). La groupalité fraternelle mise à l'épreuve par l'inceste et par la rupture familiale. Le divan familial, 1, 121136.

Granjon, E. (1987). L'enveloppe généalogique familiale. Actes du C.O.R, 73-75.

Orgiazzi, I. (1993). Rorschach et fonctionnement familial dans un cas d'anorexie mentale. Bulletin de la Société du Rorschach et des Méthodes Projectives de Langue Française, 37, 103-123.

Orgiazzi, I. (1994). Le T.A.T de l'univers familial. Bulletin de Psychologie, XLVII (416), 426-431.

Roussillon, R. (1997). Activité projective et symbolisation. Em P. Roman (Org.), Projection et symbolisation chez l'enfatn (pp. 27-35). Lyon: P.U.L.
Roman, P. (1997a). La méthode projective comme dispositif à symboliser. Em P. Roman (Org.), Projection et symbolisation chez l'enfant (pp. 37-51). Lyon: P.U.L.

Roman, P. (1997b). Troubles somatiques et catastrophes de symbolisation. Psychologie clinique et projective, 3, 75-87.

Roman, P. (1999). La position dépressive familiale: un modèle pour penser la séparation - apport des méthodes projectives. Psychiatrie de l'Enfant, XLII (1), 129-172.

Ruffiot, A. (1990). La thérapie familiale psychanalytique. Paris: Dunod.

\section{Notas:}

${ }^{1}$ La première définition de cette notion a été proposée par l'un d'entre nous (Roman, 1997b), dans le contexte de la clinique psychosomatique, pour tenter de rendre compte des modalités d'effraction des liaisons intra-psychiques dont témoigne le symptôme psychosomatique. Depuis, une reprise dans la perspective de la groupalité psychique dans le champ des épreuves projectives, permet de mettre à jour les enjeux de transmission qui sont liés au travail de la symbolisation ainsi que leur ancrage dans l'expérience somatique.

${ }^{2}$ Ces quatre figures sont présentées comme une ré-élaboration d'un premier modèle proposé précédemment (Roman, 1997)

${ }^{3}$ Il s'agit d'une pratique d'examen psychologique, au sein d'un Centre d'Action Éducative de la Protection Judiciaire de la Jeunesse (Ministère de la Justice)

${ }^{4}$ Elsa ne viendra pas au second entretien de l'examen psychologique au cours duquel la passation du T.A.T était prévue...

${ }^{5}$ Dictionnaire Larousse

\section{Sobre os autores:}

Pascal Roman: Professeur de Psychologie clinique - Centre de Recherche en Psychologie et Psychopathologie Cliniques, Institut de Psychologie, Université Lumière-Lyon 2 - E. mail: pascal.roman@ univ-lyon2.fr

Isabelle Orgiazzi Billon-Galland: Maître de Conférences en Psychologie clinique - U.F.R de Psychologie, Université P. MendèsFrance - Grenoble 2

Martine Drevon: Psychologue clinicienne, Thérapeute familiale, Chargée de cours - Institut de Psychologie, Université Lumière-Lyon 2

Michèle Chappaz: Psychologue clinicienne, Chargée de cours - U.F.R de Psychologie, Université P. Mendès-France - Grenoble 2 